\title{
Predicting MCl progression with FDG-PET and cognitive scores: a longitudinal study
}

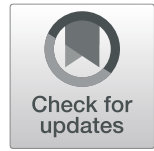

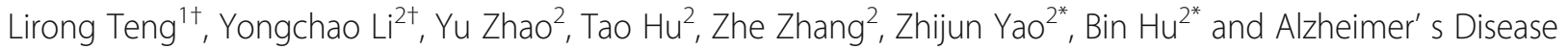
Neuroimaging Initiative (ADNI)

\begin{abstract}
Background: Mild cognitive impairment $(\mathrm{MCl})$ is an intermediate stage between normal aging and dementia. Studies on $\mathrm{MCl}$ progression are important for Alzheimer's disease (AD) prevention. 18F fluoro-deoxy-glucose positron emission tomography (FDG-PET) has been proven to be a powerful tool for measuring cerebral glucose metabolism. In this study, we proposed a classification framework for $\mathrm{MCl}$ prediction with both baseline and multiple follow-up FDG-PET scans as well as cognitive scores of 33 progressive $\mathrm{MCl}$ (pMCl) patients and 46 stable $\mathrm{MCl}(\mathrm{sMCl})$ patients from the Alzheimer's Disease Neuroimaging Initiative (ADNI).

Method: First, PET images were normalized using the Yakushev normalization procedure and registered to the Brainnetome Atlas (BNA). The average metabolic intensities of brain regions were defined as static features. Dynamic features were the intensity variation between baseline and the other three time points and change ratios with the intensity obtained at baseline considered as reference. Mini-mental State Examination (MMSE) scores and Alzheimer's disease Assessment Scale-Cognitive section (ADAS-cog) scores of each time point were collected as cognitive features. And F-score was applied for feature selection. Finally, support vector machine (SVM) with radial basis function (RBF) kernel was used for the three above features.

Results: Dynamic features showed the best classification performance in accuracy of $88.61 \%$ than static features (accuracy of 78.48\%). And the combination of cognitive features and dynamic features improved the classification performance in specificity of $95.65 \%$ and Area Under Curve (AUC) of 0.9308 .

Conclusion: Our results reported that dynamic features are more representative in longitudinal research for $\mathrm{MCl}$ prediction work. And dynamic features and cognitive scores complementarily enhance the classification performance in specificity and AUC. These findings may predict the disease course and clinical changes in individuals with mild cognitive impairment.
\end{abstract}

Keywords: FDG-PET, Mild cognitive impairment, Dynamic features, Cognitive scores, Classification

\section{Background}

Alzheimer's disease (AD), the most common form of dementia, is a progressive, irreversible and currently incurable neurodegenerative disease [1]. With the increasing of aging population, the morbidity rate of $\mathrm{AD}$ has

\footnotetext{
* Correspondence: yaozj@lzu.edu.cn; bh@lzu.edu.cn

${ }^{\dagger}$ Lirong Teng and Yongchao Li contributed equally to this work.

${ }^{2}$ Key Laboratory of Wearable Computing of Gansu Province, Lanzhou

University, Lanzhou 730000, P.R. China

Full list of author information is available at the end of the article
}

significantly increased [2]. Previous studies reported that more than 26.6 million people suffered from AD in 2006 and 1 in 85 individuals would be affected by 2050 [3]. Mild cognitive impairment (MCI) is considered a transition stage between normal aging and $\mathrm{AD}$, and conversion of patients with MCI occurs at an annualized rate of 10 to $15 \%[4,5]$. Therefore, it is vital to predict if the conditions of MCI patients would deteriorate and lead

(C) The Author(s). 2020 Open Access This article is licensed under a Creative Commons Attribution 4.0 International License, which permits use, sharing, adaptation, distribution and reproduction in any medium or format, as long as you give appropriate credit to the original author(s) and the source, provide a link to the Creative Commons licence, and indicate if changes were made. The images or other third party material in this article are included in the article's Creative Commons licence, unless indicated otherwise in a credit line to the material. If material is not included in the article's Creative Commons licence and your intended use is not permitted by statutory regulation or exceeds the permitted use, you will need to obtain permission directly from the copyright holder. To view a copy of this licence, visit http://creativecommons.org/licenses/by/4.0/ The Creative Commons Public Domain Dedication waiver (http://creativecommons.org/publicdomain/zero/1.0/) applies to the data made available in this article, unless otherwise stated in a credit line to the data. 
to $\mathrm{AD}$ within a few years, or remain stable for a long duration [6].

Neuroimaging is a powerful tool for monitoring disease progression in dementing illness [7]. Multiple studies focused on structural atrophy [8], pathological amyloid deposition [9] and metabolic alteration [10,11] to identify efficient features that can detect $\mathrm{AD}$ and MCI. In the past decades, FDG-PET which measures cerebral glucose metabolism, has been reported as an impactful MCI biomarker [12-15]. Mosconi et al. found that hypo-metabolism was indicative of potential MCI progression in the inferior parietal cortex [14]. Decreased FDG uptake was reported in posterior cingulate, temporoparietal, and prefrontal association cortex of patients with probable AD by Herholz [12]. Meanwhile, Chetelat et al. found converters had lower uptake in the right temporoparietal cortex compared with nonconverters [13]. It was also reported by Ossenkoppele et.al that FDG uptake was reduced at follow-up in the AD group in frontal, parietal and lateral temporal lobes [15]. In addition to FDG-PET, other modalities have been used, including magnetic resonance imaging (MRI) [16-20], and cerebrospinal fluid (CSF) [21-24]. Previous studies have implemented metabolic intensity of FDGPET images as features, which achieved $85.1 \%$ accuracy in classifying pMCI from sMCI at the conversion time [25] and $72.5 \%$ accuracy when combining features in baseline and 12 months follow-up [26]. M. Pagani achieved sensitivity of $92 \%$ and specificity of $91 \%$ in discriminating MCI from healthy controls when implementing metabolic differences from FDG-PET as dynamic features [27]. However, the classification performance of MCI patients needs to be improved by constructing effective classification framework.

Two study types have been applied to assess AD and $\mathrm{MCI}$, including cross-sectional and longitudinal designs. In cross-sectional studies, data for only one time point is involved (i.e., the first screening data) [21, 28-30]. At baseline, the number of subjects at different stages (i.e. $\mathrm{AD}, \mathrm{NC}$ and different kind of $\mathrm{MCI}$ ) and that of different modalities (i.e., MRI, PET, fMRI etc.) are complete. Due to greater data availability at baseline, cross-sectional data might benefit from higher statistical power. In longitudinal studies, data for multiple time points are collected, which may provide complementary information to single time point $[18,19,31-34]$. Indeed, longitudinal data can reflect the variation trend, both in structure $[18,19]$ and cognitive of individual features [32] contrasting with single time point. As $\mathrm{MCI}$ is a disease evolving over time, longitudinal data may have a great impact on its classification and detection for some lesions in the brain.

In this study, we attempted to identify progressive $\mathrm{MCI}(\mathrm{pMCI})$ and stable MCI (sMCI) using longitudinal
FDG-PET data. We first parceled FDG-PET images of each time point into 246 brain regions [35], whose average metabolic intensities were considered static features. Two types of dynamic features were defined, including intensity difference (D) between baseline and the other three time points and change ratio $(\mathrm{R})$ using the intensity at baseline as reference. Cognitive features were the MMSE scores and ADAS-cog scores of 4 time points. Then all the feature vectors were stacked to form a feature matrix. F-score was used for feature selection. Finally, leave-one-out (LOO) cross-validation was performed for classification with support vector machine (SVM). The classification framework is shown in Fig. 1.

\section{Methods}

\section{Alzheimer's disease neuroimaging initiative}

Data used in this article were obtained from the ADNI database (http://adni.loni.ucla.edu). ADNI was launched in 2003 by the National Institute on Aging, the National Institute of Biomedical Imaging and Bioengineering (NIBIB), the Food and Drug Administration (FDA), private pharmaceutical companies, and non-profit organizations, as a $\$ 60$ million,5-years public-private partnership. The subjects have been recruited from over 50 sites across the U.S. and Canada. The primary goal of ADNI is to test whether serial magnetic resonance imaging (MRI), positron emission tomography (PET), other biological markers, and clinical and neuropsychological assessment can be combined to measure the progression of $\mathrm{MCI}$ and $\mathrm{AD}$. For up-to-date information, visit www. adni-info.org.

\section{Subjects}

The general inclusion criteria for MCI are: MMSE score between 24 and 30 (inclusive) and a Clinical Dementia Rating (CDR) of 0.5 ; memory complaint; no significant levels of impairment in other cognitive domains; essential preservation of daily living activities, and absence of dementia. There are $400 \mathrm{MCI}$ subjects with more than one time-point in ADNI database. We selected subjects with 4 time points (i.e. baseline, month 6 , month 12 and month 18) FDG-PET data. Patients who converted to $\mathrm{AD}$ between baseline and 18 month was excluded, and those who converted to $\mathrm{AD}$ during 18 month to 48 month were labeled as pMCI, likewise, the patients whose situation have not changed were labeled as sMCI. Based on the criteria mentioned above, the study population comprised $46 \mathrm{sMCI}$ and $33 \mathrm{pMCI}$. The demographic and clinical information (MMSE) of all participants at baseline is shown in Table 1. The 46 sMCI patients aged $62-85$ at baseline $($ mean $=77.1 ; \mathrm{SD}=$ 6.8) (male/female, 31/15), and 33 pMCI patients aged $55-82$ at baseline (mean $=73.4 ; \mathrm{SD}=6.7) \quad($ male $/$ female, 24/9). The two groups were relatively well-matched in 


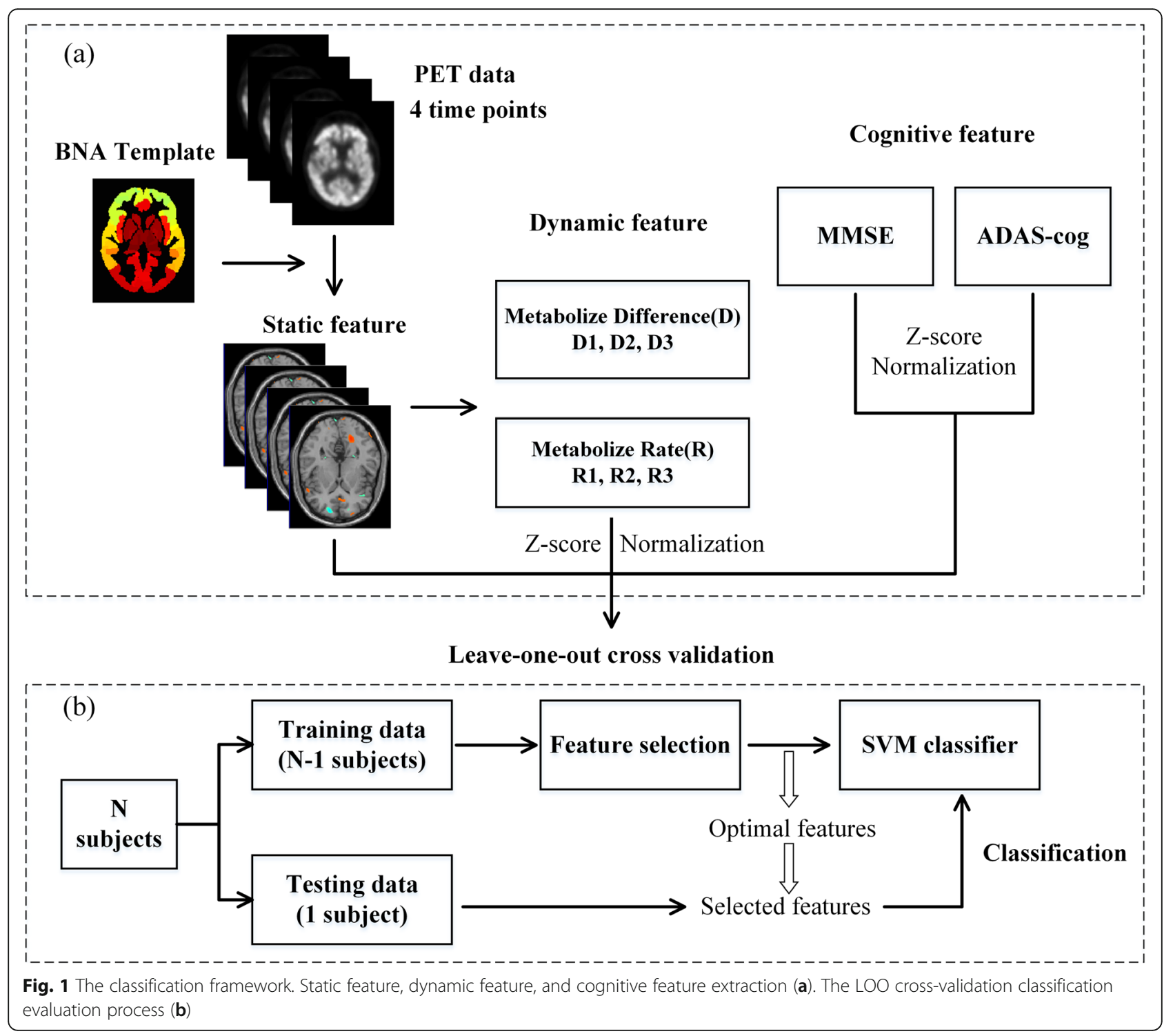

Table 1 Subject information

\begin{tabular}{llll}
\hline Group & $\mathrm{sMCl}$ & $\mathrm{pMCl}$ & $p$-value \\
\hline Number of subjects & 46 & 33 & - \\
Gender(M/F) & $31 / 15$ & $24 / 9$ & $0.6110^{\#}$ \\
Baseline age (mean \pm std) & $77.1 \pm 6.8$ & $73.4 \pm 6.7$ & $0.0192^{5^{*}}$ \\
Baseline MMSE (mean \pm std) & $27.8 \pm 1.4$ & $27.1 \pm 1.6$ & $0.0387^{5^{*}}$ \\
Baseline ADAS-cog (mean \pm std) & $13.9 \pm 5.5$ & $18.1 \pm 4.0$ & $3.86 \mathrm{e}-04^{\text {}^{*}}$
\end{tabular}

pMCI progressive mild cognitive impairment, $s M C l$ stable mild cognitive impairment, MMSE Mini-mental State Examination, ADAS-cog Alzheimer's disease Assessment Scale-Cognitive section. \# and $\$$ represent $p$-value for chisquare test and two-sample t-test, respectively. ${ }^{*}$ indicates there are significant differences of the corresponding demographic variables at baseline. terms of gender $\left(\chi^{2}=0.2590, p=0.6110\right)$. Statistic analysis indicated there were significant differences between sMCI and pMCI patients in age $(t=-2.2933, p=$ $0.0192)$, and in the demographic variables MMSE ( $\mathrm{t}=-$ 2.1036, $p=0.0387)$ and ADAS-cog $(\mathrm{t}=3.7124, p=3.86 \mathrm{e}-$ $04)$ at baseline. We implemented a linear regression to remove the effects of age and gender.

FDG-PET data acquisition and preprocessing

All FDG-PET Data were acquired using Siemens, GE and Philips PET scanners at resting-state. Details of the PET pre-processing are described at http://adni.loni.usc. edu/methods/pet-analysis/pre-processing/. First, PET images were acquired $30-60 \mathrm{~min}$ post-injection at a rate of 1 frame per $5 \mathrm{~min}$. Then, raw PET images were processed to remove the possible differences resulting from scanner differences. For a given subject, each frame was 
coregistered to the first frame, and then all frames were averaged to generate a single average image. The averaged image was reoriented and filtered into a standard $160 \times 160 \times 96$ voxel image grid with $1.5 \mathrm{~mm}$ cubic voxels. The resulting images were smoothed with $8 \mathrm{~mm}$ FWHM Gaussian kernels. Finally, all images were spatially normalized to the PET Montreal Neurological Institute (MNI) brain space template, scaled, and averaged using SPM8(Statistical Parametric Mapping 8, http://www.fil.ion.ucl.ac.uk/spm) running under Matlab 7.11(Mathworks Inc., Sherborn, MA, USA) on the CentOS 6.5.

\section{FDG-PET normalization}

Intensity normalization of FDG-PET images is often performed relative to the cerebral global mean. However, subjects with $\mathrm{AD}$ have a lower metabolic intensity than MCI across the whole brain [12, 14]. Normalization to the cerebral global mean therefore artificially scales up $\mathrm{AD}$ values while scaling down those of $\mathrm{MCI}$ cases. Yakushev et al. [36] figured out this problem between $\mathrm{AD}$ and normal control. Recent research proposed that using the signal intensity in relatively preserved regions of brain rather than the cerebral global mean value for normalization can improve group discrimination [37]. Yakushev et al. proposed a different approach for defining a reference cluster for normalization. This method consists of 2 steps. First, a cerebral global mean normalization is performed. Then, a two sample t-test is conducted in order to find the apparently hypermetabolic $(p$-value $<0.05)$ regions in the patient group compared to the healthy control, and these regions are then selected as the reference cluster. In our work, intensity normalization of the FDG-PET images was conducted performing this reference cluster method. The detailed information of the healthy control group was shown in the Supplementary table 1. From our calculation, the location of the reference clusters of different time points mainly included Precuneus, Limbic Lobe, and Posterior Cingulate. Visualization of the reference clusters of different time points was shown in Supplementary Figure 1.

\section{Feature extraction}

After preprocessing and normalization, we extracted the voxels and performed a linear regression to remove the effects of the age and gender. Then voxels were mapping into 246 regions according to the BNA template proposed by the Institute of Automation, Chinese Academy of Sciences. The BNA template is based on standard MNI space, with 210 cortical and 36 subcortical subregions, and provides a fine-grained, cross-validated atlas, containing information on both anatomical and functional connections [35]. Average metabolic intensity of regions were taken as static features.

Two types of dynamic features were defined, including intensity differences $(D)$ and the intensity change rate $(R)$. $\mathrm{D}$ is the intensity differences between baseline and the other three time points. To obtain $R$ values, the $\mathrm{D}$ values between baseline and the remaining 3 time points were then divided by the intensity of baseline. The calculation formulas are as follows:

$$
\begin{aligned}
& D_{i}=T_{\text {baseline }}-T_{i} \\
& R_{i}=\frac{D_{i}}{T_{\text {baseline }}}(i=1,2,3)
\end{aligned}
$$

$\mathrm{T}$ is the metabolic intensity of each time point. Cognitive features are the MMSE and ADAS-cog scores of 4 time points.

\section{Feature selection}

To increase the classification accuracy, effective feature selection was used for dimensionality reduction, data minimization, redundancy minimization, and calculation reduction. We applied F-score feature selection method, which shows good performance on small samples. Fscore selects the most effective features by evaluating the resolving power of the feature samples [38]. Given training samples $x_{k} \in R^{n}, \mathrm{k}=1,2, \cdots, \mathrm{l}$, and dividing the samples into positive and negative categories, the number of positive samples is $n_{+}$while the number of negative samples is $n_{-}$, then the F-score of the $\mathrm{i}$-th feature is defined as follows:

$$
F(i)=\frac{\left(\bar{x}_{i}^{(+)}-\bar{x}_{i}\right)^{2}+\left(\bar{x}_{i}^{(-)}-\bar{x}_{i}\right)^{2}}{\frac{1}{n_{+}-1} \sum_{k=1}^{n_{+}}\left(x_{k, i}^{(+)}-\bar{x}_{i}^{(+)}\right)^{2}+\frac{1}{n_{-}-1} \sum_{k=1}^{n}\left(x_{k, i}^{(-)}-\bar{x}_{i}^{(-)}\right)^{2}}
$$

$\bar{x}_{i}, \bar{x}_{i}^{(+)}$and $\bar{x}_{i}^{(-)}$are the averages of the i-th feature of the whole, positive and negative data sets, respectively. $\bar{x}_{k, i}^{+}$is the $\mathrm{i}$-th feature of the $\mathrm{k}$-th positive instance, and $\bar{x}_{k, i}^{-}$is the $\mathrm{i}$-th feature of the $\mathrm{k}$-th negative instance. The discriminating power of the feature is proportional to the $F$ value. Therefore, we can set the threshold value to exclude the features with smaller $F$ value, so as to achieve the purpose of feature selection.

In addition, LASSO feature selection method [39] was implemented to further test the stability and effectiveness of the features.

\section{Classification}

Based on the selected features above, the commonly used classifier SVM which is based on structural risk minimization and exploits a margin-based criterion was selected for classification [40, 41]. We applied LIBSVM 
library [42] on MATLAB, and the RBF kernel was utilized because of its good performance on small sample problems [43]. The RBF kernel is defined as follow:

$$
K\left(x_{1}, x_{2}\right)=\exp \left(-\frac{\left\|x_{1}-x_{2}\right\|^{2}}{2 \sigma^{2}}\right)
$$

Where $x_{1}$ and $x_{2}$ are the two feature vectors, and $\sigma$ is the width of the Gaussian kernel. To obtain a relative unbiased evaluation of classification performance, we applied the leave-one-out cross-validation strategy with feature selection and classifier training only on training set (see Fig. 1b). Specifically, one subject is first left out as testing set, and the remaining ones are used as training set. The entire process is repeated for each subject. Accuracy, sensitivity, and specificity were determined to evaluate the performance of the proposed classification framework. In addition, the Receiver Operating Characteristic (ROC) curve was used to summarize the classifier performance over a range of trade-offs between true-positive and false-positive error rates [44]. Area Under the ROC Curve (AUC) [45] was used as another measure.

\section{Results}

Feature selection results

By increasing the number of features used in classification, the optimization process of the classification results is shown in Fig. 2. When the number of features was 37, the static features in the 6th month after baseline (Static_m6) obtained the best classification performance among the static features. On the other hand, Dynamic 1 calculated with Static_mbl and Static_m6 obtained the best classification performance among the dynamic features when the number of the selected features equaled to 46 . We chose the maximum point of curve of static feature (Static m6) and dynamic feature (Dynamic 1) respectively. The common features selected from the training set at each leave-one-out were mainly located at some brain regions. We visualized these brain regions in Fig. 3. As for Static_m6, the brain regions included cingulate gyrus (average $\mathrm{F}$-score $=3.85 \mathrm{e}-5$ ), precuneus (4.49e-5), superior parietal lobule (1.00e-4), superior frontal gyrus (1.27e-4) of right hemisphere and precentral gyrus (5.61e-6), middle temporal gyrus (4.80e-5), inferior temporal gyrus (6.56e-5), precuneus (7.66e-5), inferior parietal lobule (1.59e-4), lateral occipital cortex $(1.77 \mathrm{e}-4)$ of left hemisphere. As for Dynamic_1, the brain regions mainly included precentral gyrus (3.22e-6),

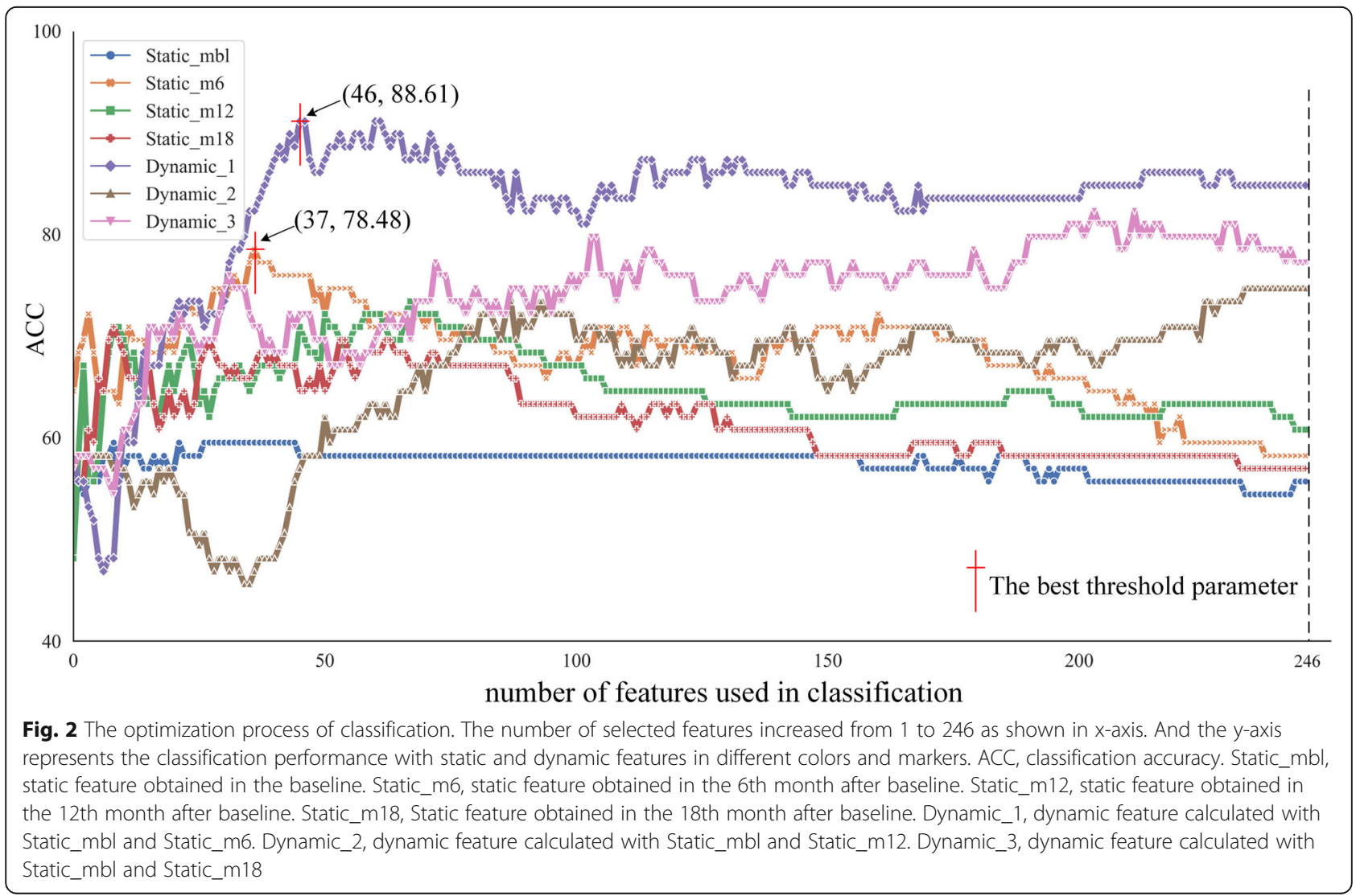




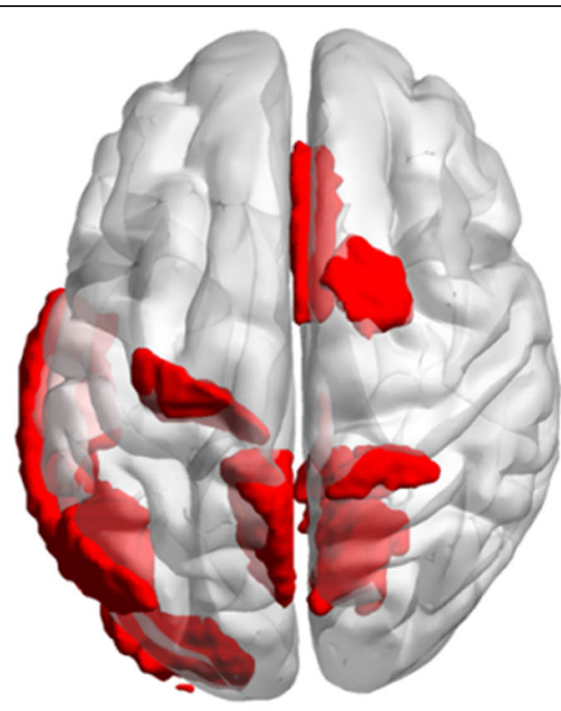

Static_m6

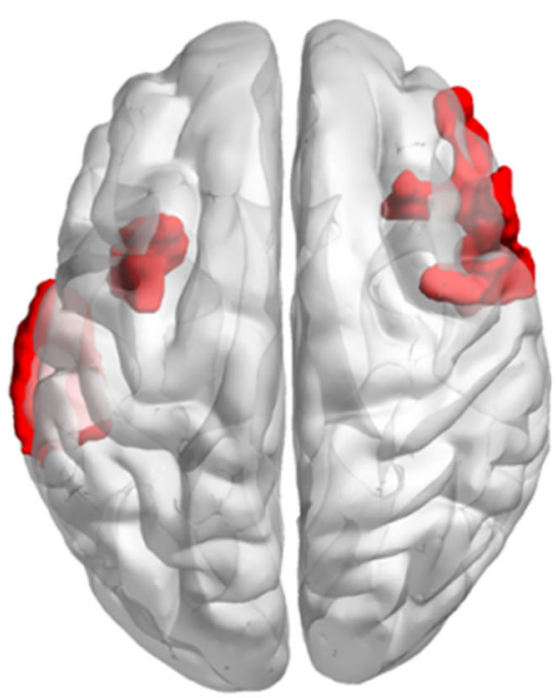

Dynamic 1

Fig. 3 Locations of the selected regions as features overlaid on the standard template. Static_m6, static feature obtained in 6 th month after baseline. Dynamic_1, dynamic feature calculated with static feature in baseline and in the 6th month after baseline

inferior frontal gyrus (5.42e-5), orbital gyrus (1.16e-4) of right hemisphere and middle temporal gyrus (1.19e-5), insular gyrus (1.86e-5) of left hemisphere.

\section{Classification results}

The classification performance was assessed based on three feature sets, which were static, dynamic and cognitive features. As shown in Tables 2 and 3 the performances of average metabolic intensity and metabolic intensity change rate were not satisfactory in classification tasks. Static feature in baseline (Static_mbl) achieved accuracy of $59.49 \%$, sensitivity of $6.06 \%$ specificity of $97.83 \%$ and AUC of 0.5402 . Static features in the 6th month after baseline (Static_m6) achieved accuracy of $78.48 \%$, sensitivity of $57.58 \%$, specificity of $93.48 \%$ and

Table 2 Comparison of the classification performance in static features

\begin{tabular}{lllll}
\hline Feature & ACC(\%) & SEN(\%) & SPE(\%) & AUC \\
\hline Static_mbl & 59.49 & 6.06 & 97.83 & 0.5402 \\
Static_m6 & 78.48 & 57.58 & 93.48 & 0.6634 \\
Static_m12 & 73.41 & 48.48 & 91.30 & 0.6344 \\
Static_m18 & 70.88 & 45.45 & 89.13 & 0.5428 \\
Static_all & 75.95 & 51.52 & 93.48 & 0.6614 \\
\hline
\end{tabular}

ACC classification accuracy, SEN classification sensitivity, SPE classification specificity, AUC Area Under Curve, Static_mbl static feature obtained in the baseline, Static_m6 static feature obtained in the 6th month after baseline, Static_m12 static feature obtained in the 12th month after baseline, Static $m 18$ static feature obtained in the 18th month after baseline, Static all combining all the static features
AUC of 0.6634. Meanwhile, in the third time point (12th month after baseline, Static_m12), static features achieved accuracy of $73.41 \%$, sensitivity of $48.48 \%$, specificity of $91.30 \%$ and AUC of 0.6344 . Accuracy of $70.88 \%$, sensitivity of $45.45 \%$, specificity of $89.13 \%$, and AUC of 0.5428 were obtained by implementing static features in the 18th month after baseline (Static_m18). We also combined all static features, but the results did not meet expectations. The combined accuracy was lower than Static_m6 but higher than other three time points. As for dynamic features, the intensity change rates did not

Table 3 Comparison of the classification performance in dynamic features

\begin{tabular}{lllll}
\hline Feature & ACC(\%) & SEN(\%) & SPE(\%) & AUC \\
\hline Dynamic_1 & $\mathbf{8 8 . 6 1}$ & $\mathbf{8 1 . 8 2}$ & $\mathbf{9 3 . 4 8}$ & $\mathbf{0 . 9 3 5 1}$ \\
Dynamic_2 & 77.21 & 75.76 & 78.26 & 0.8063 \\
Dynamic_3 & 82.28 & 73.91 & 93.94 & 0.9289 \\
R1 & 60.76 & 6.32 & 100 & 0.8524 \\
R2 & 64.56 & 15.22 & 100 & 0.6278 \\
R3 & 64.56 & 54.55 & 71.74 & 0.5738 \\
Dynamic_all & 87.38 & 87.88 & 86.96 & 0.8959 \\
\hline
\end{tabular}

ACC classification accuracy, SEN classification sensitivity, SPE classification specificity. AUC Area Under Curve, Dynamic_1 dynamic feature calculated with Static_mbl and Static_m6 in Table 2, Dynamic_2 dynamic feature calculated with Static_mbl and Static_m12,Dynamic_3 dynamic feature calculated with Static_mbl and Static_m18, $R 1$ metabolic change rate in the 6th month after baseline, $R 2$ metabolic change rate in the 12 th month after baseline, $R 3$ metabolic change rate in the 18th month after baseline, Dynamic_all combining all the dynamic features 


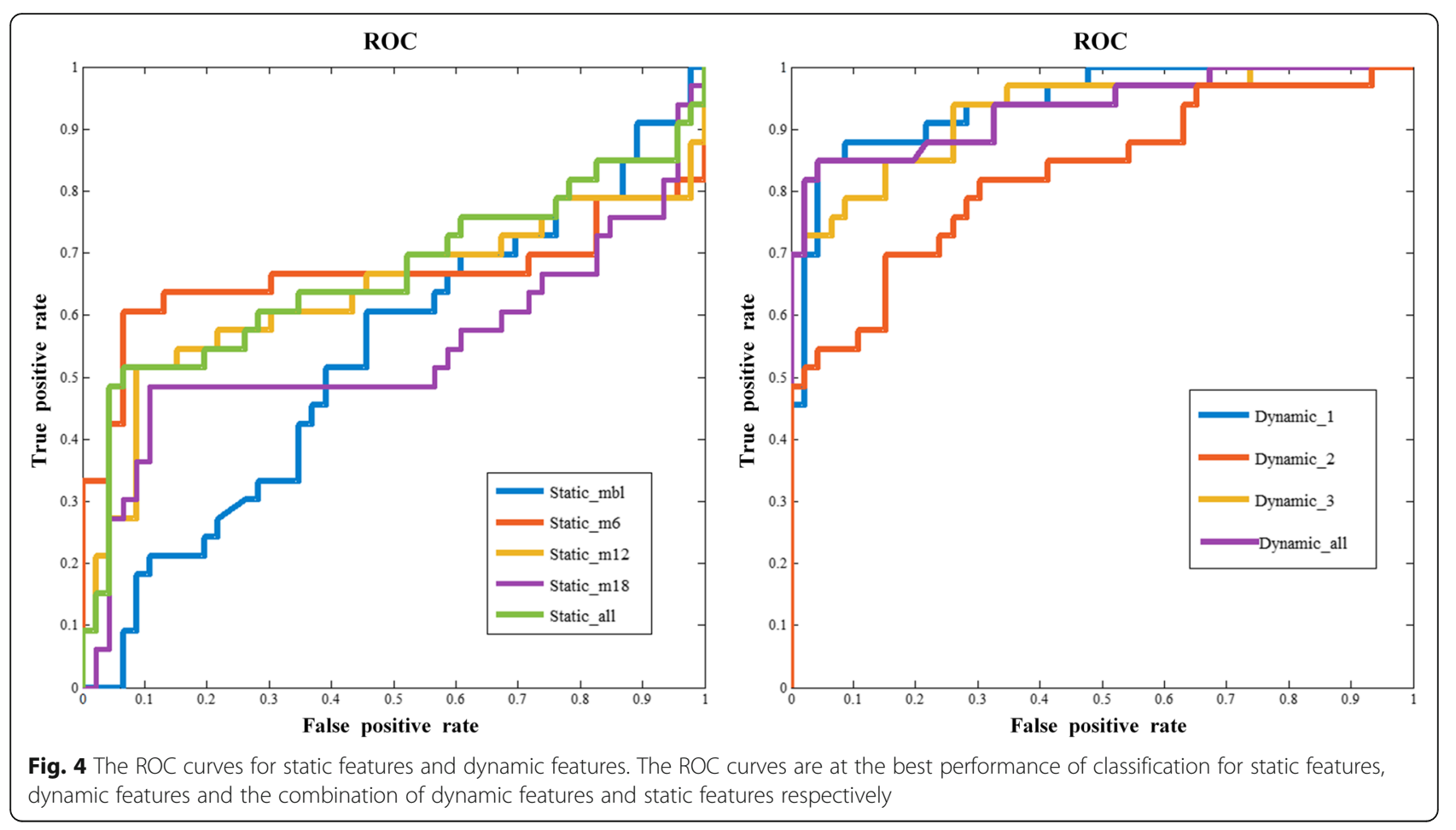

shown good classification effect, but the metabolic differences Dynamic_1 calculated by Static_mbl and Static m6 got the highest accuracy of $88.61 \%$ which is better than the static features. All the ROC curves were shown in Fig. 4. In addition, LASSO feature selection based classification results were shown in supplementary table 2 and supplementary table 3. Under LASSO method, Static_m6 obtained the best classification performance with accuracy of $75.94 \%$, sensitivity of $60.61 \%$, specificity of $80.43 \%$, and AUC of $75.96 \%$. As for dynamic features, Dynamic_1 achieved accuracy of $87.34 \%$, sensitivity of 93.94\%, specificity of 82.61, and AUC of 0.9302. Compared with LASSO based classification results, F-score

Table 4 Comparison of the classification performance in the combination features

\begin{tabular}{lllll}
\hline Feature & ACC(\%) & SEN(\%) & SPE(\%) & AUC \\
\hline Static & 75.95 & 51.52 & 93.48 & 0.6614 \\
Dynamic & $\mathbf{8 7 . 3 8}$ & $\mathbf{8 7 . 8 8}$ & $\mathbf{8 6 . 9 6}$ & $\mathbf{0 . 8 9 5 9}$ \\
Cognitive & 77.22 & 55.55 & 93.48 & 0.6414 \\
Static \& Dynamic & 72.15 & 57.58 & 82.61 & 0.7444 \\
Static \& Cognitive & 73.42 & 57.58 & 84.78 & 0.7345 \\
Dynamic \& Cognitive & 87.34 & 75.76 & 95.65 & 0.9308 \\
All & 72.42 & 36.36 & 97.83 & 0.6443
\end{tabular}

ACC classification accuracy, SEN classification sensitivity, SPE classification specificity, AUC Area Under Curve. Static denotes the combination of all the static features, which is the same as Static_all in Table 2. Dynamic denotes the combination of all the dynamic features as shown in Table 3 (Dynamic_all). Cognitive denotes the combination of all the MMSE and ADAS-cog score features. All denotes the combination of all the types of features based results showed better classification performance both in static features and in dynamic features.

Table 4 shows the feature combination results respectively. Combining static features of the 4 time points, an accuracy of $75.95 \%$ was obtained which exceeded more than $5 \%$ compared with single time point except M6, and the sensitivity and specificity were also improved to 51.52 and $93.38 \%$. Accuracy of combined dynamic features reached $87.38 \%$, higher than that of static features. Cognitive features got a specificity of $93.48 \%$.

\section{Discussion}

The present study developed a classification framework using both cross-sectional and longitudinal FDG-PET as well as cognitive scores to discriminate pMCI from sMCI. We adequately considered effects from static, dynamic and cognitive features. All these types of features were compared to ensure accurate relationship measurement. Our findings suggested that the dynamic features outperformed previous studies of pMCI and SMCI classification, with an accuracy of $88.61 \%$ by SVM RBF [19, $31,46,47]$.

Cerebral glucose metabolism measured by FDG-PET is an impactful mean of MCI prediction. Metabolic intensity reflects integrated synaptic activity. Decreased metabolic intensity in a given brain region indicates either reduced number of synapses or decreased synaptic metabolic activity [48]. Sensitive biomarkers were selected in each type of features, according to Fig. 4, as the middle temporal lobe, cingulate gyrus, inferior frontal 
gyrus, orbital gyrus, parahippocampal gyrus and postcentral gyrus. In previous studies, many of these selected regions have been indicated as significant in the conversion prediction for MCI patients[49-51].

\section{Classification performance evaluation}

Table 2 demonstrated that combination of all static features yielded an accuracy of $75.95 \%$, achieving better performance in discriminating pMCIs from sMCIs than any single time point except the 6th month after baseline. In this study, result biasing random classification was obtained at baseline. We inferred that the baseline is too far from the disease transformation time point, with little effect on the classification. However, at the early stage of MCI conversion (after baseline), the classification accuracy decreased with time. Therefore, we believed that in the early stage of $\mathrm{MCI}$, the disease deteriorated rapidly. As the disease progresses, the conversion rate slows down. In Table 3, this speculation was further confirmed: Dynamic_1 obtained the highest accuracy and Dynamic_2, Dynamic_3 was lower. As Table 3 shown, Dynamic_all achieved a better prediction accuracy than Dynamic_2, and Dynamic_3 with 10.17 and $5.10 \%$ increment respectively. But Dynamic_3 performs better in specificity of $93.94 \%$.

Several studies combined static and dynamic features in MCI classification or prediction [19, 31]. Gray et al. obtained an accuracy of $63.1 \%$ while combining longitudinal changes with 12-month FDG-PET signal intensities, with 58.4 and $62.3 \%$ for baseline and 12-month signal intensities [31]. Thung et al. combined baseline and 18-month MRI volumetric and dynamic features, and achieved an accuracy of $78.2 \%$, which is $6.6 \%$ higher than when using only the reference time point [19]. As shown in Table 4, combination of all the dynamic features achieved the better classification accuracy of $87.38 \%$ than the other feature combinations. On the one hand, combining dynamic features and static features did not get a better classification accuracy. But the sensitivity (57.58\%) and the AUC (0.7444) was improved compared with the combination of static features (sensitivity of 51.52\% and AUC of 0.6614). On the other hand, combining dynamic features and cognitive features achieved better classification performance (accuracy of $87.34 \%$, sensitivity of $75.76 \%$, specificity of $95.65 \%$ and AUC of 0.9308 ) than the combination of cognitive features (accuracy of $77.22 \%$, sensitivity of $55.55 \%$, specificity of $93.48 \%$ and AUC of 0.6414$)$. The present results indicated that the dynamic features can provide some complementary information which can enhance classification performance in conjunction with the static features and cognitive features respectively. Additionally, compared with all the static (sensitivity of $51.52 \%$ and AUC of 0.6614) and all the cognitive features (sensitivity of $55.55 \%$ and AUC of 0.6414 ), classification performance was improved in sensitivity of $57.58 \%$ and AUC of 0.7345 with the combination of static features and cognitive features. This above result showed that static features and cognitive features assistant to each other when predicting pMCIs from sMCIs. When applying all the dynamic, static and cognitive features, classification performance in specificity was improved to $97.83 \%$.

However, it should be noted that the combination of all the features performs worse than some specific combinations. On the one hand, this maybe suffered from the limitations of F-score feature selection method. A disadvantage of F-score is that it does not consider mutual information among features [38]. Despite F-score showed effectiveness on dynamic features, F-score might lack ability in selecting features with complementary information from different feature sets, as static features and cognitive features. On the other hand, the poor performance of the feature combination may due to the over determination or lack of convergence of SVM classifier.

Multiple studies also examined the contribution of cognitive scores [32, 52]. For instance, Cui et al. used different modalities of data, including neuropsychological and functional measures, to explore the optimal set of predictors of conversion from $\mathrm{MCI}$ to $\mathrm{AD}$, and obtained an accuracy of $67.13 \%$ [52]. Zhang et al. reported that the combination of cognitive scores (MMSE and ADAS-cog) can improve the accuracy, sensitivity, and specificity in distinguishing $\mathrm{MCI}$ and $\mathrm{AD}$ [32]. In the current study, the combination of MMSE and ADAS$\operatorname{cog}$ scores of 4 time points resulted in lower accuracy of $77.22 \%$ and higher specificity of $93.48 \%$. And the combination of cognitive features and static features showed in Table 4 got higher sensitivity of $57.58 \%$ and AUC of 0.7345 . Also, the combination of cognitive features and dynamic features showed in Table 4 achieved better specificity of $95.65 \%$ and AUC of 0.9308 .

As shown above, the $88.61 \%$ classification accuracy was achieved while using the dynamic features in Dynamic_1 (Table 3). Sensitivity $(87.88 \%$, in Table 4$)$ improved by dynamic features and specificity $(95.65 \%$, in Table 4) increased by cognitive features strongly contributed to the remarkable results obtained, suggesting that longitudinal data and cognitive scores complementarily enhance the classification performance.

\section{Methodological limitations}

The limitations of this study should be mentioned. First, the combination of multimodal data has been shown to improve the classification results in multiple studies [28, $32,47,52,53]$. Other modality data also have different sensitive biomarkers which are complementary in enhancing discrimination performance. In addition, more 
effective dynamic feature computing methods need to be proposed in the future study.

\section{Conclusions}

Our study suggested that dynamic features got the best classification accuracy than the static features and features of cognitive scores in discriminating pMCIs from sMCIs. And dynamic features and cognitive scores complementarily enhance the classification performance in sensitivity and specificity. Furthermore, the brain regions related to the selected dynamic features might suggest the different progression patterns between pMCIs and sMCIs.

\section{Supplementary information}

Supplementary information accompanies this paper at https://doi.org/10. 1186/s12883-020-01728-X.

Additional file 1.

\section{Abbreviations}

FDG-PET: 18 Fluoro-deoxy-glucose positron emission tomography; AD: Alzheimer's disease; MCl: Mild cognitive impairment; $\mathrm{pMCl}$ : progressive $\mathrm{MCl}$; sMCl: stable MCl; pMCls: pMCl patients; sMCls: sMCl patients; ADNI: Alzheimer's Disease Neuroimaging Initiative; BNA: Brainnetom Atlas; MMSE: Mini-mental State Examination; ADAS-cog: Alzheimer's disease Assessment Scale-Cognitive section; RBF: Radial bias function; MRI: Magnetic resonance imaging; CFS: Cerebrospinal fluid; SVM: Support vector machine; CDR: Clinical Dementia Rating; MNI: Montreal Neurological Institute; ROC: Receive Operating Characteristic; AUC: Area Under the ROC Curve

\section{Acknowledgments}

Data collection and sharing for this project was funded by the Alzheimer's Disease Neuroimaging Initiative (ADNI) (National Institutes of Health Grant U01 AG024904) and DOD ADNI (Department of Defense award number W81XWH-12-2-0012). ADNI is funded by the National Institute on Aging, the National Institute of Biomedical Imaging and Bioengineering, and through generous contributions from the following: AbbVie, Alzheimer's Association; Alzheimer's Drug Discovery Foundation; Araclon Biotech; BioClinica, Inc.; Biogen; Bristol-Myers Squibb Company; CereSpir, Inc.; Cogstate; Eisai Inc.; Elan Pharmaceuticals, Inc.; Eli Lilly and Company; Eurolmmun; F. Hoffmann-La Roche Ltd. and its affiliated company Genentech, Inc.; Fujirebio; GE Healthcare; IXICO Ltd.; Janssen Alzheimer Immunotherapy Research \& Development, LLC.; Johnson \& Johnson Pharmaceutical Research \& Development LLC.; Lumosity; Lundbeck; Merck \& Co., Inc.; Meso Scale Diagnostics, LLC.; NeuroRx Research; Neurotrack Technologies; Novartis Pharmaceuticals Corporation; Pfizer Inc.; Piramal Imaging; Servier; Takeda Pharmaceutical Company; and Transition Therapeutics. The Canadian Institutes of Health Research is providing funds to support ADNI clinical sites in Canada. Private sector contributions are facilitated by the Foundation for the National Institutes of Health (www.fnih.org). The grantee organization is the Northern California Institute for Research and Education, and the study is coordinated by the Alzheimer's Therapeutic Research Institute at the University of Southern California. ADNI data are disseminated by the Laboratory for Neuro Imaging at the University of Southern California.

\section{Authors' contributions}

$L T, Y L, Y Z, Z Y$ and $B H$ conceived and designed the experiments. $L T, Y L, Y Z$, and $T H$ organized and analyzed the raw data. $L T, Z Y, Y Z, Z Z$, and $T H$ participated in the statistical analysis and interpretation of data. $L T, Y L$, and $\mathrm{YZ}$ wrote the manuscript, and $\mathrm{BH}, \mathrm{TH}, \mathrm{ZZ}$, and $\mathrm{ZY}$ polished the manuscript. All author(s) read and approved the final manuscript.

\section{Authors' information}

Data used in preparation of this article were obtained from the Alzheimer's Disease Neuroimaging Initiative (ADNI) database (adni.loni.usc.edu). As such, the investigators within the ADNI contributed to the design and implementation of ADNI and/or provided data but did not participate in analysis or writing of this report. A complete listing of ADNI investigators can be found at: http://adni.loni.usc.edu/wp-content/uploads/how_to_apply/ ADNI_Acknowledgement_List.pdf

\section{Funding}

This work was supported in part by the National Natural Science Foundation of China (Grant No.61632014, No.61627808, No.61210010), in part by the National Basic Research Program of China (973 Program) under Grant 2014CB744600, in part by the Program of Beijing Municipal Science \& Technology Commission under Grant Z171100000117005 and in part by the Fundamental Research Funds for the Central Universities (Izujbky-2018-it64). All these funds provided computing resources for this study.

\section{Availability of data and materials}

We obtained permission to access ADNI repository from http://adni.loni.usc. edu/data-samples/access-data/. For readers, login credentials are required to access the ADNI datasets. Readers can easily download the dataset used in this study according to our supplementary file, the Subject-ID.txt once they obtained permission to access ADNI repository. Alternatively, they can contact the corresponding authors by E-mail to get the preprocessed data used in this study.

Ethics approval and consent to participate

Not applicable.

\section{Consent for publication}

Not applicable.

\section{Competing interests}

The authors declare that they have no competing interests.

\section{Author details}

${ }^{1}$ Department of Obstetrics and Gynecology, Peking Union Medical College, Chinese Academy of Medical Sciences, Beijing 100032, P.R. China. ${ }^{2}$ Key Laboratory of Wearable Computing of Gansu Province, Lanzhou University, Lanzhou 730000, P.R. China.

Received: 20 March 2019 Accepted: 14 April 2020

Published online: 21 April 2020

\section{References}

1. Winblad B, et al. Defeating Alzheimer's disease and other dementias: a priority for European science and society. Lancet Neurol. 2016;15(5):455-532.

2. Patterson C. World Alzheimer report 2018: the state of the art of dementia research: new frontiers. London: Alzheimer's Disease International; 2018.

3. Brookmeyer R, et al. Forecasting the global burden of Alzheimer's disease. Alzheimers Dement. 2007;3(3):186-91.

4. Morris JC. And J.J.J.o.a.s.D. Cummings, Mild cognitive impairment (MCI) represents early-stage Alzheimer's disease. J Alzheimers Dis. 2005;7(3):235-9.

5. Galimberti D. And E.J.J.o.n. Scarpini, Progress in Alzheimer's disease. J Neurol. 2012;259(2):201-11.

6. Prince M, Bryce R, Ferri C. World Alzheimer Report 2011: The benefits of early diagnosis and intervention; 2018.

7. Veitch DP, et al. Understanding disease progression and improving Alzheimer's disease clinical trials: recent highlights from the Alzheimer's disease neuroimaging initiative. Alzheimers Dement. 2019;15(1):106-52.

8. Edmonds $\mathrm{EC}$, et al. Heterogeneous cortical atrophy patterns in $\mathrm{MCl}$ not captured by conventional diagnostic criteria. Neurology. 2016;87(20):210816

9. Palmqvist $\mathrm{S}$, et al. Detailed comparison of amyloid PET and CSF biomarkers for identifying early Alzheimer disease. Neurology. 2015;85(14):1240-9.

10. Cerami C, et al. Brain metabolic maps in mild cognitive impairment predict heterogeneity of progression to dementia. Neuroimage Clin. 2015;7:187-94.

11. Toledo JB et al. Metabolic network failures in Alzheimer's disease: a biochemical road map. Alzheimers Dement. 2017;13(9):965-84. 
12. Herholz K, et al. Discrimination between Alzheimer dementia and controls by automated analysis of multicenter FDG PET. Neuroimage. 2002;17(1):30216.

13. Chetelat $\mathrm{G}$, et al. Mild cognitive impairment: can FDG-PET predict who is to rapidly convert to Alzheimer's disease? Neurology. 2003;60(8):1374-7.

14. Mosconi $\mathrm{L}$, et al. $\mathrm{MCl}$ conversion to dementia and the APOE genotype: a prediction study with FDG-PET. Neurology. 2004;63(12):2332-40.

15. Ossenkoppele $R$, et al. Longitudinal imaging of Alzheimer pathology using [11 C] PIB,[18 F] FDDNP and [18 F] FDG PET. Eur J Nucl Med Mol Imaging. 2012;39(6):990-1000

16. Willette AA, et al. Prognostic classification of mild cognitive impairment and Alzheimer's disease: MRI independent component analysis. Psychiatry Res Neuroimaging. 2014;224(2):81-8.

17. Li F, et al. A robust deep model for improved classification of $\mathrm{AD} / \mathrm{MCl}$ patients. IEEE J Biomed Health Inform. 2015;19(5):1610-6.

18. Ma X, et al. Identify the atrophy of Alzheimer's disease, mild cognitive impairment and normal aging using morphometric MRI analysis. Front Aging Neurosci. 2016;8:243

19. Thung $\mathrm{K}-\mathrm{H}$, et al. Identification of progressive mild cognitive impairment patients using incomplete longitudinal MRI scans. Brain Struct Funct. 2016; 221(8):3979-95.

20. Liu K, et al. Prediction of mild cognitive impairment conversion using a combination of independent component analysis and the cox model. Front Hum Neurosci. 2017;11:33.

21. Davatzikos C, et al. Prediction of MCl to AD conversion, via MRI, CSF biomarkers, and pattern classification. Neurobiol Aging. 2011;32(12):2322.e19-27.

22. Gray KR, et al. Random forest-based similarity measures for multi-modal classification of Alzheimer's disease. Neuroimage. 2013;65:167-75.

23. Popp J, et al. Cerebrospinal fluid cortisol and clinical disease progression in $\mathrm{MCl}$ and dementia of Alzheimer's type. Neurobiol Aging. 2015;36(2):601-7.

24. Forlenza OV, et al. Cerebrospinal fluid biomarkers in Alzheimer's disease: diagnostic accuracy and prediction of dementia. Alzheimers Dement. 2015; 1(4):455-63.

25. Cabral $\mathrm{C}$, et al. Predicting conversion from $\mathrm{MCl}$ to $\mathrm{AD}$ with FDG-PET brain images at different prodromal stages. Comput Biol Med. 2015;58:101-9.

26. Nozadi SH, Kadoury S, A.s.D.N. Initiative. Classification of Alzheimer's and $\mathrm{MCl}$ patients from semantically parcelled PET images: a comparison between AV45 and FDG-PET. Int J Biomed imaging. 2018;2018:1247430.

27. Pagani $\mathrm{M}$, et al. Volume of interest-based [18F] fluorodeoxyglucose PET discriminates $\mathrm{MCl}$ converting to Alzheimer's disease from healthy controls. A European Alzheimer's disease consortium (EADC) study. Neurolmage Clin. 2015;7:34-42.

28. Hinrichs $C$, et al. Predictive markers for $A D$ in a multi-modality framework: an analysis of $\mathrm{MCl}$ progression in the ADNI population. Neuroimage. 2011 55(2):574-89.

29. Ye J, et al. Sparse learning and stability selection for predicting $M C l$ to $A D$ conversion using baseline ADNI data. BMC Neurol. 2012;12(1):46.

30. Weiner MW, et al. The Alzheimer's disease neuroimaging initiative: a review of papers published since its inception. Alzheimers Dement. 2013:9(5):e111-94.

31. Gray KR, et al. Multi-region analysis of longitudinal FDG-PET for the classification of Alzheimer's disease. Neuroimage. 2012;60(1):221-9.

32. Zhang D, Shen D, A.S.D.N.IJ.P. one. Predicting future clinical changes of MCl patients using longitudinal and multimodal biomarkers. PLoS One. 2012;7(3):e33182.

33. Rodrigues F, Silveira M. Longitudinal FDG-PET features for the classification of Alzheimer's disease. Proceedings of the 2014 36th Annual International Conference of the IEEE Engineering in Medicine and Biology Society, EMBC 2014. 2014(2014):1941-4.

34. Zhan Y, et al. Longitudinal study of impaired intra-and inter-network brain connectivity in subjects at high risk for Alzheimer's disease. J Alzheimers Dis. 2016;52(3):913-27.

35. Fan $L$, et al. The human brainnetome atlas: a new brain atlas based on connectional architecture. Cereb Cortex. 2016:26(8):3508-26.

36. Yakushev I, et al. Choice of reference area in studies of Alzheimer's disease using positron emission tomography with fluorodeoxyglucose-F18. Psychiatry Res Neuroimaging. 2008;164(2):143-53.

37. Yakushev I, et al. SPM-based count normalization provides excellent discrimination of mild Alzheimer's disease and amnestic mild cognitive impairment from healthy aging. Neuroimage. 2009:44(1):43-50.

38. Chen Y.-W, Lin C.-J. Combining SVMs with various feature selection strategies. Feature Extraction and Applications (Studies in Fuzziness and Soft Computing). Germany: Springer Berlin/Heidelberg. 2006. p. 315-24.
39. Fonti V, Belitser E. Feature selection using lasso. VU Amsterdam Research Paper in Business Analytics; 2017. p. 1-25.

40. Tax D, de Ridder D, Duin RP. Support vector classifiers: a first look. In: Proceedings ASCl; 1997.

41. Vapnik V. SVM method of estimating density, conditional probability, and conditional density. In: 2000 IEEE International Symposium on Circuits and Systems. Emerging Technologies for the 21st Century. Proceedings (IEEE Cat No. 00CH36353). Chicago: IEEE. 2000;2:749-52

42. Chang C-C, C.-J.J.A.t.o.i.s. Lin, and Technology. LIBSVM: a library for support vector machines. ACM Trans Intell Syst Technol. 2011;2(3):27.

43. Hertz $T$, Hillel $A B$, Weinshall D. Learning a kernel function for classification with small training samples. Proceedings of the 23rd international conference on Machine learning, Pittsburgh, PA. 2006. p. 401-8.

44. Swets JAJS. Measuring the accuracy of diagnostic systems. Science. 1988; 240(4857):1285-93.

45. Bradley AP. The use of the area under the ROC curve in the evaluation of machine learning algorithms. Pattern Recogn. 1997:30(7):1145-59.

46. Moradi E, et al. Machine learning framework for early MRI-based Alzheimer's conversion prediction in MCI subjects. Neuroimage. 2015;104:398-412.

47. Xu L, et al. Multi-modality sparse representation-based classification for Alzheimer's disease and mild cognitive impairment. Comput Methods Prog Biomed. 2015:122(2):182-90.

48. Rocher $A B$, et al. Resting-state brain glucose utilization as measured by PET is directly related to regional synaptophysin levels: a study in baboons. Neuroimage. 2003;20(3):1894-8.

49. Zhang $X$, et al. Resting-state whole-brain functional connectivity networks for $\mathrm{MCl}$ classification using L2-regularized logistic regression. IEEE Trans Nanobiosci. 2015;14(2):237-47.

50. Wang $W-Y$, et al. Impacts of CD33 genetic variations on the atrophy rates of hippocampus and parahippocampal gyrus in normal aging and mild cognitive impairment. Mol Neurobiol. 2017;54(2):1111-8.

51. Lin F, et al. Insula and inferior frontal gyrus' activities protect memory performance against Alzheimer's disease pathology in old age. J Alzheimers Dis. 2017;55(2):669-78

52. Cui $Y$, et al. Identification of conversion from mild cognitive impairment to Alzheimer's disease using multivariate predictors. PLoS One. 2011;6(7): e21896.

53. Wang $P$, et al. Multimodal classification of mild cognitive impairment based on partial least squares. J Alzheimers Dis. 2016:54(1):359-71.

\section{Publisher's Note}

Springer Nature remains neutral with regard to jurisdictional claims in published maps and institutional affiliations.

Ready to submit your research? Choose BMC and benefit from:

- fast, convenient online submission

- thorough peer review by experienced researchers in your field

- rapid publication on acceptance

- support for research data, including large and complex data types

- gold Open Access which fosters wider collaboration and increased citations

- maximum visibility for your research: over $100 \mathrm{M}$ website views per year

At BMC, research is always in progress.

Learn more biomedcentral.com/submissions 